\title{
Carnets
}

Revue électronique d'études françaises de l'APEF

Première Série - 2 Numéro Spécial 10-11 | 2011

D'un Nobel l'autre

\section{L'actualité de Sartre sur la scène portugaise}

\section{Ana Clara Santos}

\section{OpenEdition}

\section{Journals}

Édition électronique

URL : http://journals.openedition.org/carnets/5683

DOI : 10.4000/carnets.5683

ISSN : 1646-7698

Éditeur

APEF

Édition imprimée

Date de publication : 1 janvier 2011

Pagination : 175-187

Référence électronique

Ana Clara Santos, «L'actualité de Sartre sur la scène portugaise », Carnets [En ligne], Première Série - 2 Numéro Spécial 10-11 | 2011, mis en ligne le 16 juin 2018, consulté le 01 mai 2019. URL : http:// journals.openedition.org/carnets/5683 ; DOI : 10.4000/carnets.5683

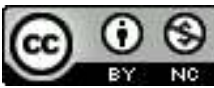

Carnets est mis à disposition selon les termes de la licence Creative Commons - Atribution - Pas d'utilisation commerciale 4.0 International. 


\title{
L'ACTUALITÉ DE SARTRE SUR LA SCÈNE PORTUGAISE
}

ANA ClaRA SANTOS

Université d'Algarve

anaclaravsantos@gmail.com

\begin{abstract}
Résumé
Jean-Paul Sartre, prix Nobel de la littérature en 1964, a été le premier auteur à décliner, de plein gré, la distinction de l'académie suédoise: "Aucun artiste, aucun écrivain, aucun homme ne mérite d'être consacré de son vivant, parce qu'il a le pouvoir et la liberté de tout changer." Ce sentiment de liberté et d'indépendance absolue de la pensée et de la création a fait de lui, on le sait, l'un des écrivains les plus controverses de son temps et un classique incontournable du XXe siècle. Distingué par une œuvre dont l'esprit de liberté et la recherche de la vérité symbolise la vaste influence sur notre époque, Sartre garde encore toute son actualité. Ses écrits littéraires et philosophiques en langue portugaise chez plusieurs éditeurs au moment de la dictature continuent à être réédités. Ses pièces de théâtre, jouées et traduites au moment de la censure, continuent à attirer l'attention des artistes portugais et brésiliens au début du XXle siècle. Huis-clos, La putain respectueuse et Les Troyennes constituent un trio édificateur sur la scène portugaise car il s'agit bien pour les artistes portugais d'élever un modèle dramatique sur l'engagement de l'individu dans une méditation sur le tragique et la condition humaine.
\end{abstract}

\begin{abstract}
Jean-Paul Sartre, awarded the Nobel prize in Literature in 1964, was the first author to willingly decline the distinction of the Swedish academy: "No artist, no writer, no man deserves to be consecrated in his lifetime, because he has the power and the freedom to change everything." This feeling of freedom and absolute independence of thought and creation, made him, as we know, one of the most controversial writers of his time and a major classic of the 20th century. Distinguished by a work in which the spirit of freedom and the search for the truth symbolize a major influence of our time, Sartre's work remains up-to-date. His literary and philosophic works translated into Portuguese and published through several editors at the time of the dictatorship continue to be republished. His plays, played and translated at the time of the censorship, continue to draw the attention of the Portuguese and Brazilian artists at the beginning of the 21 st century. Huis-clos, La putain respectueuse and Les Troyennes establish an edifying trio on the Portuguese stage because it is indeed a question for the Portuguese artists of raising a dramatic model on the commitment of the individual to a meditation of the tragic and the human condition.
\end{abstract}

Mots-clés: Sartre, prix Nobel, réception, censure, actualité, Portugal

Keywords: Sartre, Nobel prize, reception, censorship, present, Portugal 
L'écrivain est en situation dans son époque. Sartre, Les Temps Modernes

\section{Sartre et le prix Nobel}

Le prix Nobel institué par testament par le chimiste suédois Alfred Nobel qui devait distinguer un lauréat, auteur d'une "œuvre littéraire faisant preuve d'un puissant idéal”, est devenu la récompense la plus prestigieuse et la plus médiatique à l'échelle planétaire. Ce prix décerné depuis 1901 à des écrivains du monde entier a récompensé souvent des œuvres littéraires en langue française. On compte, entre le prix attribué, en 1901, à Sully Prudhomme et celui décerné à Jean-Marie Le Clézio, en 2008, 15 lauréats en français ${ }^{1}$. Or s'il est vrai que ce prix contribue à la reconnaissance d'une œuvre et la singularité d'un projet d'écriture, il n'est pas moins vrai que certains écrivains vont décliner ce prix et cette distinction. Certains y seront obligés comme c'est le cas de Boris Pasternak, en 1958, sous la pression politique du gouvernement russe. Certains y renonceront de plein gré. Tel est le cas de Jean-Paul Sartre qui refuse le prix de l'Académie suédoise décerné, le 22 octobre 1964, "pour son œuvre qui par l'esprit de liberté et la recherche de la vérité dont elle témoigne a exercé une vaste influence sur notre époque"2. Son refus du prix Nobel, en 1964, allait de pair avec la déclinaison de la Légion d'Honneur, en 1945, ou celle d'une chaire au Collège de France. Voici un extrait de la lettre qu'il envoya à l'Académie suédoise:

Je regrette vivement que l'affaire ait pris une apparence de scandale: un prix est distribué et quelqu'un le refuse. [...] J'y ai invoqué deux sortes de raisons: des raisons personnelles et des raisons objectives. Les raisons personnelles sont les suivantes: mon refus n'est pas un acte improvisé. J'ai toujours décliné les distinctions officielles. [...] Ce n'est pas la même chose si je signe Jean Paul Sartre ou si je signe Jean Paul Sartre prix Nobel. [...] L'écrivain doit donc refuser de se laisser transformer en institution même si cela a lieu sous les formes les plus honorables comme c'est le cas. [...] Mes raisons objectives sont les suivantes: le seul combat actuellement possible sur le front de la culture est celui pour la coexistence pacifique des deux cultures, celles de l'est et celle de l'ouest. Je ne veux pas dire qu'il faut qu'on s'embrasse, je sais bien que la confrontation entre ces deux cultures doit nécessairement prendre la forme d'un conflit, mais elle doit avoir lieu entre les hommes et entre les cultures, sans intervention des institutions. [...] Mes sympathies vont indéniablement au socialisme et à ce qu'on appelle le bloc de l'est, mais je suis né et j'ai été élevé dans une famille bourgeoise. [...] J'espère cependant bien entendu

\footnotetext{
${ }^{1}$ À titre d'exemple, cela représente $14,7 \%$ contre $26,4 \%$ pour les lauréats en langue anglaise.

${ }^{2}$ Ces propos ont été proférés lors de l'annonce du prix Nobel.
} 
que "le meilleur gagne ", c'est à dire le socialisme. C'est pourquoi je ne peux accepter aucune distinction distribué par les hautes instances culturelles, pas plus à l'est qu'à l'ouest, même si je comprends fort bien leur existence ${ }^{3}$.

Ce refus du Prix Nobel a été aussi, on le voit, une forme d'illustration du partage entre Sartre philosophique et Sartre homme politique. Institutionnalisés, ces honneurs auraient fait de lui, dans sa perspective, un instrument figé, aliéné de sa liberté. II le réitère quelques années plus tard:

J'ai refusé le Prix Nobel de littérature parce que je refusais que l'on consacre Sartre avant sa mort. Aucun artiste, aucun écrivain, aucun homme ne mérite d'être consacré de son vivant, parce qu'il a le pouvoir et la liberté de tout changer. Le Prix Nobel m'aurait élevé sur un piédestal alors que je n'avais pas fini d'accomplir des choses, de prendre ma liberté et d'agir, de m'engager. Tout acte aurait été futile après, puisque déjà reconnu de façon rétrospective. Imaginez: un écrivain pourrait recevoir ce prix et se laisser aller à la déchéance, tandis qu'un autre pourrait devenir encore meilleur. Lequel des deux méritait son prix? Celui qui était au sommet et qui a redescendu la pente ou celui qui fut consacré avant d'atteindre le sommet? J'aurais pu être l'un des deux, et jamais personne n'aurait pu prédire ce que je ferais. On est ce que l'on fait. Je ne serai jamais récipiendaire du Prix Nobel, tant et aussi longtemps que je pourrai encore agir en le refusant ${ }^{4}$.

\section{Sartre traduit en portugais}

La culture portugaise a su rendre hommage à l'œuvre sartrienne et a reçu ses idées philosophiques, ses écrits littéraires et ses pièces dramatiques avec enthousiasme. Lors des commémorations du centenaire de sa naissance, plusieurs colloques ont été organisés, notamment à Lisbonne et à Porto ${ }^{5}$. II faut dire qu'il avait été accueilli de son vivant dans cet espace qui lui a rendu hommage en 2005 . En

\footnotetext{
${ }^{3}$ II faut dire que ce n'était pas la première lettre qu'il adressa à l'Académie, car des mois auparavant, face à la possibilité de se voir décerné le prix, il envoya la missive suivante: "Monsieur le Secrétaire, D'après certaines informations dont j'ai eu connaissance aujourd'hui, j'aurais cette année quelques chances d'obtenir le prix Nobel. Bien qu'il soit présomptueux de décider d'un vote avant qu'il ait eu lieu, je prends à l'instant la liberté de vous écrire pour dissiper ou éviter un malentendu. Je vous assure d'abord, Monsieur le secrétaire, de ma profonde estime pour l'académie suédoise et le prix dont elle a honoré tant d'écrivains. Toutefois pour des raisons qui me sont personnelles et pour d'autres qui sont plus objectives, je désire de ne pas figurer sur la liste des lauréats possibles et je ne peux ni ne veux, ni en 1964, ni plus tard, accepter cette distinction honorifique". Toutes les références ici citées peuvent être consultées en ligne sur http://vietsciences.free.fr/nobel/litterature/sartre.htm.

${ }^{4}$ Idem, ibidem.

${ }^{5}$ On fait référence ici au colloque organisé par Cassiano Reimão, à l'Universidade Nova de Lisboa (UNL), intitulé "Jean-Paul Sartre, uma cultura da alteridade: filosofia e literatura" et à celui co-organisé par Cristina Marinho et José Almeida, à la Faculté de Lettres de l'université de Porto (FLUP), subordonné au thème "Sartre, um filósofo na literatura".
} 
effet, un an après la révolution des œillets, il est invité, au début du mois d'avril 1975, par Arnaldo Saraiva et Isabel Pires de Lima, à l'université de Porto, à la suite de sa visite à l'usine en autogestion Sousa e Abreu à Guimarães ${ }^{6}$. Au moment de la dictature, malgré la censure qui pesait sur ces œuvres au Portugal, il a toujours fait l'objet d'une profonde admiration au sein de l'intelligentsia nationale qui continuait à le lire, en langue française, clandestinement. Le monde éditorial a contribué, par ailleurs, au Brésil et au Portugal, à la diffusion de son œuvre en langue portugaise. Comme on peut observer dans le tableau qui suit, grand nombre de ses œuvres ont été traduites et rééditées par trois grandes maisons d'édition nationales, EuropaAmérica, Presença et Bertrand, au cours des deux dernières décennies de la dictature de Salazar, entre 1958 et 1974.

\begin{tabular}{|c|c|c|c|c|}
\hline Date & Titre & Traducteur & Éditeur & Rééditions \\
\hline 1941 & $\begin{array}{l}\text { As estátuas } \\
\text { volantes }\end{array}$ & $\begin{array}{l}\text { Adolfo Casais } \\
\text { Monteiro }\end{array}$ & Inquérito & \\
\hline 1951 & $\begin{array}{l}\text { O diabo e o bom } \\
\text { Deus }\end{array}$ & $\begin{array}{l}\text { Gabriela } \\
\text { Alves Neves }\end{array}$ & $\begin{array}{l}\text { Editores } \\
\text { Associados }\end{array}$ & 1976 \\
\hline 1958 & A Náusea & $\begin{array}{l}\text { António } \\
\text { Coimbra } \\
\text { Martins }\end{array}$ & $\begin{array}{l}\text { Europa-América } \\
\text { Público }\end{array}$ & $\begin{array}{l}1965\left(3^{a}\right), 1969,1973 \\
1976,1990 \\
2003\end{array}$ \\
\hline $\begin{array}{l}1960 \\
\left(2^{\underline{a}}\right)\end{array}$ & $\begin{array}{l}\text { Reflexões sobre o } \\
\text { racismo }\end{array}$ & $\begin{array}{l}\text { Difusão } \\
\text { Europeia do } \\
\text { Livro }\end{array}$ & $1968\left(5^{\underline{a}}\right)$ & \\
\hline 1960 & As mãos sujas & $\begin{array}{l}\text { António } \\
\text { Coimbra } \\
\text { Martins }\end{array}$ & Europa-América & $\begin{array}{l}\text { 1962, 1965, 1972, } \\
1982\end{array}$ \\
\hline 1960 & Acuso & $\begin{array}{l}\text { Mário T. } \\
\text { Alves, Gaspar } \\
\text { Barbosa }\end{array}$ & Brasília & 1969 \\
\hline 1961 & $\begin{array}{l}\text { A prostituta } \\
\text { respeitosa }\end{array}$ & Miroel Silveira & & \\
\hline 1961 & $\begin{array}{l}\text { Os sequestrados } \\
\text { de Altona }\end{array}$ & $\begin{array}{l}\text { António } \\
\text { Coimbra } \\
\text { Martins }\end{array}$ & Europa-América & 1963, 1973 \\
\hline 1961 & Kean & $\begin{array}{l}\text { Fernando } \\
\text { Midões }\end{array}$ & Presença & \\
\hline
\end{tabular}

\footnotetext{
${ }^{6}$ Rappelons, au passage, que ce contact avec la culture portugaise donna lieu à un projet de publication intitulé "Portugal aujourd'hui" dans sa revue Les Temps Modernes. II l'annonça dans une interview publiée par la revue Árvore (no 1, mai 1975): “Je pense qu'en réalité il est important de parler un peu de ce qu'on a vu ici, et surtout il est important de pousser les portugais à le dire. On a l'intention de publier un numéro des Temps Modernes, avec la collaboration des portugais, qui sera dédié à "Portugal aujourd'hui".
} 


\begin{tabular}{|c|c|c|c|c|}
\hline 1961 & $\begin{array}{l}\text { Mortos sem } \\
\text { sepultura }\end{array}$ & $\begin{array}{l}\text { Francisco da } \\
\text { Conceição }\end{array}$ & Presença & 1965,1974 \\
\hline 1962 & $\begin{array}{l}\text { O existencialismo é } \\
\text { um humanismo }\end{array}$ & $\begin{array}{l}\text { Vergílio } \\
\text { Ferreira }\end{array}$ & $\begin{array}{l}\text { Presença } \\
\text { Bertrand }\end{array}$ & $\begin{array}{l}\text { 1970, 1972, } 1978 \\
2004\end{array}$ \\
\hline 1962 & As Moscas & Nuno Valadas & Presença & $\begin{array}{l}\text { 1965, 1974, 1979, } \\
198-, 1983,1986\end{array}$ \\
\hline 1963 & $\begin{array}{l}\text { Os dados estão } \\
\text { lançados }\end{array}$ & $\begin{array}{l}\text { Maria Luísa } \\
\text { Vieira Rosa }\end{array}$ & Presença & $\begin{array}{l}1971,1972,1976, \\
1983\end{array}$ \\
\hline 1963 & A idade da razão & Sérgio Millet & Bertrand & $1975,1981\left(4^{a}\right)$ \\
\hline 1964 & Situações VI & $\begin{array}{l}\text { Valentina } \\
\text { Trigo Sousa }\end{array}$ & Europa-América & 1975 \\
\hline 1965 & Situações VII & $\begin{array}{l}\text { Valentina } \\
\text { Trigo Sousa }\end{array}$ & Europa-América & 1976 \\
\hline 1965 & $\begin{array}{l}\text { Com a morte na } \\
\text { alma }\end{array}$ & Sérgio Millet & $\begin{array}{l}\text { Difusão Europeia } \\
\text { do Livro }\end{array}$ & $1966,1968\left(4^{\mathrm{a}}\right)$ \\
\hline 1965 & $\begin{array}{l}\text { Esboço de uma } \\
\text { teoria das emoções }\end{array}$ & $\begin{array}{l}\text { A.Pastor } \\
\text { Fernandes, } \\
\text { João Lopes } \\
\text { Alves }\end{array}$ & Presença & 1972 \\
\hline 1966 & As Troianas & $\begin{array}{l}\text { Rolando } \\
\text { Roque da } \\
\text { Silva }\end{array}$ & $\begin{array}{l}\text { Difusão Europeia } \\
\text { do Livro }\end{array}$ & \\
\hline 1966 & As Palavras & J. Guinsburg & Bertrand & $\begin{array}{l}\text { 1967, 1971, } 1973, \\
1974,1979,1982\end{array}$ \\
\hline 1968 & Situações I & $\begin{array}{l}\text { Rui Mário } \\
\text { Gonçalves }\end{array}$ & Europa-América & 1976 \\
\hline 1968 & Situações III & $\begin{array}{l}\text { Rui Mário } \\
\text { Gonçalves }\end{array}$ & Europa-América & 1971 \\
\hline 1968 & Situações IV & $\begin{array}{l}\text { Eduardo } \\
\text { Prado Coelho }\end{array}$ & Europa-América & \\
\hline 1968 & Situações V & & T. Brasileiro & \\
\hline 1971 & $\begin{array}{l}\text { O escritor não é } \\
\text { político? }\end{array}$ & $\begin{array}{l}\text { António } \\
\text { Pescada, } \\
\text { António Serra, } \\
\text { Guilherme } \\
\text { Valente }\end{array}$ & D. Quixote & \\
\hline 1972 & A engrenagem & $\begin{array}{l}\text { Sousa } \\
\text { Victorino }\end{array}$ & Presença & $1980\left(3^{a}\right)$ \\
\hline $\begin{array}{l}197 \\
1976 \\
\left(2^{a}\right)\end{array}$ & $\begin{array}{l}\text { Os caminhos da } \\
\text { liberdade }\end{array}$ & Sérgio Millet & Bertrand & 1979, 1981, 1983 \\
\hline 1973 & As Troianas & $\begin{array}{l}\text { Helena } \\
\text { Cidade Moura }\end{array}$ & Plátano & \\
\hline
\end{tabular}




\begin{tabular}{|c|c|c|c|c|}
\hline 1974 & $\begin{array}{l}\text { O diabo e o bom } \\
\text { Deus }\end{array}$ & $\begin{array}{l}\text { Serafim } \\
\text { Ferreira }\end{array}$ & $\begin{array}{l}\text { Círculo de } \\
\text { Leitores }\end{array}$ & \\
\hline 1975 & Pena suspensa & $\begin{array}{l}\text { Amélia } \\
\text { Petinga }\end{array}$ & Bertrand & $1982\left(4^{a}\right)$ \\
\hline 1975 & $\begin{array}{l}\text { Com a morte na } \\
\text { alma }\end{array}$ & Isabel de Brito & Bertrand & $1985\left(4^{a}\right)$ \\
\hline 1977 & Situações $X$ & Pedro Támen & António Ramos & \\
\hline 1977 & $\begin{array}{l}\text { Entre quatro } \\
\text { paredes }\end{array}$ & & Victor Civita & \\
\hline 1984 & $\begin{array}{l}\text { Cadernos de } \\
\text { Guerra }\end{array}$ & $\begin{array}{l}\text { Manuela } \\
\text { Torres, Carlos } \\
\text { Araújo }\end{array}$ & Difel & 1985 \\
\hline 1988 & A imaginação & $\begin{array}{l}\text { Manuel João } \\
\text { Gomes }\end{array}$ & Difel & 2002 \\
\hline 1993 & O Ser e o nada & $\begin{array}{l}\text { G. Cascais } \\
\text { Franco }\end{array}$ & $\begin{array}{l}\text { Círculo de } \\
\text { Leitores }\end{array}$ & \\
\hline
\end{tabular}

\section{Sartre joué sur la scène portugaise}

Du point de vue théâtral, la dramaturgie sartrienne marque son entrée en territoire national au cours des années 40 d'une façon emblématique car elle coïncide avec la première tentative dramaturgique de l'écrivaine Natália Correia. La pièce Huis-clos, traduite et jouée clandestinement par Natália Correia auprès d'un cercle restreint d'amis ${ }^{7}$ constitue effectivement, à notre connaissance, la première concrétisation de la dramaturgie sartrienne sur la scène portugaise ${ }^{8}$. Par la suite, cette pièce fera l'objet de nombreuses expériences dramaturgiques au sein du théâtre amateur, du théâtre scolaire et du théâtre expérimental ${ }^{9}$. Elle sera jouée à nouveau le 3 juillet 1978 sous le titre Porta fechada par le Grupo Teatro Hoje d'après une traduction de Fiama Hasse Pais Brandão et la mise en scène de Jorge Listopad. S'il faut attendre, par la suite, le mois de mars $1990^{10}$ et le mois d'avril $1995^{11}$ pour que des projets de théâtre s'intéressent à la pièce, il faut reconnaître qu'au début du XXle

\footnotetext{
${ }^{7}$ II faut dire que la pièce compta avec la mise en scène de Carlos Wallenstein, la scénographie de João Santiago et les interprétations de Natália Correia, Maria Ferreira, Alexandre Castro Freire et Manuel de Lima. Le ํㅡㄴ 35 du journal Portugal llustrado rend hommage à cette initiative culturelle à laquelle sont venues de nombreuses figures de la vie artistique, intellectuelle, mondaine et diplomatique portugaise telles que Francisco Sousa Tavares, João Gaspar Simões, Ruben Leitão, Isabel da Nóbrega et les écrivains Almada Negreiros, Urbano Tavares Rodriques et Sofia de Mello Breyner, entre autres.

${ }^{8}$ Rappelons que très peu d'années séparent la création de la pièce à Paris au théâtre du Vieux Colombier datée du 27 mai 1944 (mise en scène par Raymond Rouleau) et la représentation à Lisbonne chez Natália Correia.

${ }_{9}$ Pour plus de détails, consulter l'annexe 1.

${ }^{10}$ Cette pièce, traduite par Matilde Antunes, est insérée dans un projet du groupe Arte Viva, projecto Teatro e Comunicação du Barreiro et est jouée, d'après une mise en scène de Jorge Cardoso qui combine le texte de Sartre avec celui de José Régio, le 31 mars 1990, à l'Externato Manuel de Mello (Barreiro).

${ }_{11}$ Huis-clos, à porta fechada est un projet du groupe groupe Cenadro, Centro de Artes Performativas joué à l'école secondaire prof. Herculano de Carvalho à Olivais. Cette mise en scène de Fernando Oliveira, jouée, tout d'abord, du 28 avril au 8 mai, est rejouée à la Sociedade Filarmónica Recordação de Apolo, à Lisbonne, du 3 au 26 juin 1995.
} 
siècle Huis-clos connaît un nouvel essor auprès de différentes troupes théâtrales professionnelles. Le 22 juin 2001, elle est mise en scène par Kot Kotecki, toujours sous le titre $A$ porta fechada, au Théâtre Expérimental du Funchal (TEF) au Théâtre municipal Baltazar Dias à Madère. Le 17 février 2008, ce sera au tour du Grupo de Teatro O Grito de reprendre la pièce, cette fois-ci sous le titre Huis-clos (Sem saída), dans le cadre de la $12^{\mathrm{e}}$ édition de la Mostra de Teatro de Almada, au Forum municipal Romeu Correia (Auditório Fernando Lopes Graça, Almada) ${ }^{12}$. Le 21 octobre 2009, Jorge Andrade de la troupe Mala Voadora met en scène la pièce, sous le titre Huis-clos, en réponse à l'illustration d'un cycle consacré aux prérogatives et aux limitations sociales et artistiques de l'identité (O Negócio ZDB, Lisbonne, du 21 au 27 octobre).

Contrairement à Huis-clos, les pièces Les séquestrés d'Altona (1959) et Les jeux sont faits (1947) ont connu uniquement une mise en scène chacune: le 20 septembre 1979, Os sequestrados de Altona fut jouée par le Teatro Popular - Companhia Nacional 1 et le 29 mai 1986, ce fut au tour de Os dados estão lançados par le Grupo de Teatro Esteiros. La pièce Les Mouches a attiré, à son tour, l'attention des troupes universitaires. Elle fut choisie par les étudiants de $3^{\mathrm{e}}$ année de l'Escola Superior de Teatro e Cinema et fut jouée, du 4 au 8 juin 1997, au Teatro Nacional D. Maria II, à Lisbonne, dans une mise en scène signée par Carlos J. Pessoa. La troupe théâtrale universitaire de l'université d'Algarve, Sin-Cera, joua As moscas lors de la $5^{\mathrm{e}}$ édition des Rencontres de Théâtre Universitaire au Teatro Académico Gil Vicente à Coimbra. Cette mise en scène de Pedro Wilson sera reprise au Teatro Lethes à Faro, le 28 janvier 2004, et le 23 mai, au Teatro Municipal Maria Matos dans le cadre de la $5^{\mathrm{e}}$ édition du festival annuel du théâtre académique de Lisbonne.

La Prostituée respectueuse bénéficia d'une fortune assez surprenante en langue portugaise. En effet, elle est jouée à Paris, au théâtre Antoine, le 8 novembre 1946 et, deux ans plus tard, elle était jouée en langue portugaise. Tout d'abord, au Brésil, dans une mise en scène présentée au théâtre Fénix, à Rio de Janeiro, le 5 décembre 1948. Le rôle de la protagoniste a été créé par Olga Navarro et repris, ensuite, par Maria Della Costa au Teatro Popular de Arte de Sandro Polloni. Après une tournée au Brésil, celle-ci viendra, en 1957, en tournée au Portugal ${ }^{13}$. En 1961, la traduction de la pièce a fait l'objet d'une publication au Brésil qui a circulé aussi au Portugal. Les éditeurs justifient la circulation de la pièce par son caractère actualisant du mélodrame et sa remise en cause d'un système social et politique décadents:

\footnotetext{
${ }^{12}$ Comme on peut voir dans l'annexe 1 , cette mise en scène fut jouée à nouveau le 20 mars 2008 dans la $3^{\mathrm{e}}$ édition de la Mostra de Teatro de Alverca dans l'Espaço Cegada et, enfin, au Teatro Extremo à Almada, le 10 septembre 2009.

${ }^{13}$ Remarquons au passage que la représentation de la troupe brésilienne est censurée et interdite à Porto.
} 
Dans la Prostituta Respeitosa qu'on publie maintenant en livre et qu'une bonne partie du public connaît déjà des représentations théâtrales, ou de l'adaptation cinématographique, le thème apparent est celui de la ségrégation raciale aux EtatsUnis - problème terrible et tragique que cette nation procure peser à travers des lois et des processus éducationnels. Mais, en vérité, celui-ci sert uniquement de toile de fond au drame particulier de Lizzie, la prostituée, qui découle du drame fondamental de notre époque: le conflit de base entre deux schémas de vie, le capitalisme et le socialisme $^{14}$ (Silveira, 1961).

La pièce sera jouée ensuite par la troupe du Teatro Experimental do Porto (TEP), le 18 décembre 1998, dans une mise en scène de Norberto Barroca ${ }^{15}$. À propos de cette représentation portugaise de $A$ Respeitosa, Carlos Porto renforce, dans sa critique du spectacle publiée dans le Jornal de Letras, le caractère extrêmement actuel de la pièce sartrienne:

Encore plus évidente dans les temps qui courent où le sexe domine et est dominé par la société et la politique nord-américaine, alors que le racisme continue présent dans l'une et dans l'autre. Pièce politique située entre le crime raciste et la corruption, entre la fausse morale et le royaume du mensonge (Porto, 1999).

Mais c'est sans doute son adaptation des Troyennes d'Euripide qui a atteint le plus grand succès auprès du public portugais. Cette pièce a été jouée, pour la première fois, le 17 novembre 1994, par le Projecto Teatral, Associação Cultural, d'après la traduction et la mise en scène de Maria Duarte et Elsa Valentim, au Centro Cultural de Belém dans le cadre du festival biannuel de jeunes créateurs d'Europe et de la Méditerranée. Quinze mois plus tard, João Mota accepte de revenir au Théâtre National D. Maria II, cette fois-ci comme metteur en scène, à la condition de pouvoir choisir une pièce: Les Troyennes. Or il est pour le moins étonnant que son choix retombe non pas sur le modèle grec, mais sur le modèle français, celui des Troyennes de Sartre d'après la traduction éditée en 1973 par Helena Cidade Moura $^{16}$. Le public portugais découvre, le 15 février 1996, grâce aux grands acteurs de la troupe nationale tels qu’Eunice Muñoz, António Rama, João Grosso, Madalena Braga, Mafalda Vilhena, Manuela Couto, São José Lapa, Fernando Serafim et Helena Vieira une

\footnotetext{
${ }^{14}$ La traduction de cet extrait des "Notes des éditeurs" a été faite par nous-mêmes, tout comme toutes les autres traductions du portugais insérées dans cet article.

${ }^{15}$ La pièce, traduite par Norberto Barroca et Mário Dias Garcia, a été jouée, tout d'abord, au mois de décembre 1998, au Clube Fenianos Portuenses et, ensuite, à l'Auditório municipal de Vila Nova de Gaia, le 9 décembre 2000.

${ }^{26}$ II faut dire que cette traduction de l'adaptation sartrienne a fait l'objet du premier numéro de la collection "Teatro Vivo" de l'édition Plátano qui prétendait, à travers la publication de textes inédits, diffuser les grands classiques de la dramaturgie contemporaine afin de constituer une histoire vivante du théâtre.
} 
pièce au féminin qui, selon le metteur en scène, offre une vision du monde martyrisé par la guerre:

Je n'ai pas oublié la guerre d'Angola, je n'ai pas oublié Timor, je n'ai pas oublié la Bosnie, je n'ai pas oublié les milliers de guerres en devenir. [...] Le choix de l'adaptation de Sartre et non pas de l'original se doit au fait que cet Auteur et surtout l'Homme a été très important dans ma formation et, ensuite, parce que sa réécriture d'Euripide nous conduit à éviter l'archéologie et à rencontrer l'essence vitale de la tragédie. Une fois encore le cercle: la mère-terre, le ventre de la mère, le Destin, les dieux, le Cosmos (Mota 1996: 9-10).

Pour l'artiste portugais, cette adaptation des Troyennes de Jean-Paul Sartre se situe donc entre le texte d'Euripide et celui de l'auteur français dans une volonté de préservation de l'écoute originelle d'un chant lyrique qui se fait entendre comme un "oratorio" institué, à côté de l'espace viril de la politique et de la guerre, comme l'espace féminin et des voix du deuil. Ces mécanismes ont été mis en place pour dramatiser la pièce afin que le public du XXè siècle puisse ressentir, comme disait Sartre, "les vérités profondes exprimées par Euripide". Si la réécriture du mythe sous la plume de Sartre soulève, avant tout, comme l'a si bien démontré la critique, la question de l'avènement des régimes colonialistes en tant qu'arme de prévention et de réveil des consciences, il n'en reste pas moins évident que les manifestations du deuil féminin sont devenues la seule arme d'un combat désarmé et sans issue dans une méditation sur le tragique et la condition humaine.

Deux ans plus tard, le 3 octobre 1998, la troupe Contacto présente à nouveau la pièce dans le cadre de la $15^{\mathrm{e}}$ édition du festival de théâtre amateur d'Évora. On le voit, les grandes troupes théâtrales professionnelles et les troupes de théâtre amateur ont bien succombé sous le charme de cette forme lyrique et emblématique de la pièce exploitée auparavant par le metteur en scène grec, Michel Cacoyannis, responsable de la mise en scène au Théâtre National Populaire ${ }^{17}$ (Palais Chaillot, le 10 mars 1965) et à New York:

La vision finale des ruines de Troie avec toutes les femmes déportées et l'assassinat du jeune Astyanax est suffisamment claire pour évoquer le souvenir de n'importe quel génocide, ancien ou récent. II revient au public d'associer le génocide à telle ou telle image, à telle ou telle mémoire.

Bernard Pingaud a voulu comprendre, dans une interview que Jean-Paul Sartre lui a accordé en février 1965, quelques jours avant la première, cet intérêt de philosophe envers

\footnotetext{
${ }^{17}$ Rappelons que la pièce compta alors avec l'interprétation de Jean Martinelli, Françoise Le Bail, Éléonore Hirt, Jean-Pierre Bernard, Judith Magre, Nathalie Nerval, Yves Vincent et Françoise Brion.
} 
la tragédie "la plus statique, la moins théâtrale qui soit". Pourquoi s'inspirer de ce "beau monument en ruine qu'on visite avec respect, sous la conduite d'exégètes scrupuleux, mais que personne n'aurait l'idée d'habiter", de ce théâtre si "loin de nous" inspiré "d'une conception religieuse du monde qui nous est devenue complètement étrangère"? La réponse de Sartre est une vraie leçon sur l'art théâtral qui soulève non seulement les questions de la distinction entre traduction/adaptation ${ }^{18}$ et celle de la déconstruction (ce qu'il appelle "décomposition"), mais aussi la problématique de la nécessité d'une distance qui puisse mener à la réflexion. Pour lui, le drame de l'absurde d'un Beckett ou d'un lonesco a accompli la même révolution que la tragédie d'Euripide par rapport à la tragédie d'Eschyle:

Les Troyennes ont été représentées pendant la guerre d'Algérie, dans une traduction très fidèle de Jacqueline Moatti. J'avais été frappé du succès qu'avait obtenu ce drame auprès d'un public favorable à la négociation avec le F.L.N. C'est évidemment cet aspect du drame qui m'a intéressé d'abord. Vous n'ignorez pas que, du temps même d'Euripide, il avait une signification politique précise. Il était une condamnation de la guerre en général, et des expéditions coloniales en particulier. La guerre, nous savons aujourd'hui ce que cela signifie: une guerre atomique ne laissera ni vainqueurs ni vaincus. C'est précisément ce que toute la pièce démontre: les Grecs ont détruit Troie, mais ils ne tireront aucun bénéfice de leur victoire puisque la vengeance des Dieux les fera périr tous. Que "tout homme sensé doive éviter la guerre", comme l'affirme Cassandre, il n'était même pas besoin de le dire: la situation des uns et des autres en témoigne assez, J'ai préféré laisser à Poséidon le mot de la fin: "Vous en crèverez tous" (Sartre 1966).

En tant qu'auteur "engagé", il lui fallait opérer dans un terrain neutre, qui ne soit ni à l'est ni à l'ouest, mais qui puisse toucher un public global, loin de l'institutionnalisation de la littérature, aussi bien les élites que le peuple:

Que signifie la littérature dans un monde qui a faim? Comme la morale, la littérature a besoin d'être universelle. L'écrivain doit donc se ranger aux côtés du plus grand nombre, des deux milliards d'affamés, s'il veut pouvoir s'adresser à tous et être lu par tous.

[...] Je voulais montrer comment un homme peut passer de la littérature considérée comme sacrée à une action qui reste néanmoins celle d'un intellectuel [...] Ce que je lui demande c'est de ne pas ignorer la réalité et les problèmes qui se posent [...]

\footnotetext{
18 "Entre la tragédie d'Euripide et la société athénienne du Ve siècle existe un rapport implicite que nous ne pouvons plus voir aujourd'hui que du dehors. Si je veux rendre ce rapport sensible, je ne peux donc pas me contenter de traduire la pièce, il faut que je l'adapte. Un langage de pure imitation était exclu; la transposition en français parlé moderne l'était également, car le texte doit aussi marquer sa propre distance par rapport à nous. J'ai donc choisi un langage poétique, qui garde au texte son caractère cérémonieux, sa valeur rhétorique - mais qui en modifie l'accent".
} 
Voyez-vous, l'écrivain contemporain doit écrire à travers ses malaises en essayant de les élucider (Sartre, 1964).

Que l'importation du modèle sartrien corresponde à une visée philosophique ou politique, elle a bien trouvé sa voie, dans les mains des artistes portugais, en pleine consonance avec la pensée sartrienne, au sein d'une conduite vers l'engagement de l'individu, l'interrogation sur le sens de l'action et le rejet du conformisme. 


\section{Bibliographie}

MotA, João (1996). "As Troianas", in Teatro Nacional D. Maria II, As Troianas. Eurípides. Jean-Paul Sartre. Lisboa: TNDMII.

PORTO, Carlos, "Demasiado respeitosos", in Jornal de Letras, 13.1.1999.

SARTRE, Jean-Paul (1964). Le Monde, 10 juillet.

SARTRE, Jean-Paul (1966). Les Troyennes. Paris: Gallimard.

SILVEIRA, Miroel (1961). Sartre. A prostituta respeitosa, peça em um acto e dois quadros. Rio de Janeiro: Ed. Civilização Brasileira, "Biblioteca do leitor moderno", vol. 23.

Sites consultés:

http://vietsciences.free.fr/nobel/litterature/sartre.htm

http://expositions.bnf.fr/sartre/ 


\section{Annexe - Représentations de Sartre au Portugal}

- 1957 - Respeitosa: Companhia de Maria Della Costa [Brasil]

- 3/7/1978 - Porta fechada: Grupo Teatro Hoje

- 20/9/1979 - Os sequestrados de Altona: Teatro Popular - Companhia Nacional 1

- 29/5/1986 - Os dados estão lançados: Grupo de Teatro Esteiros

-31/3/1990 - Embora os teus olhos sejam: Arte Viva, Companhia de Teatro do Barreiro

-17/11/1994 - As troianas: Projecto Teatral

- 28/4/1995 - Huis-clos, à porta fechada: Cenadro

-15/2/1996 - As troianas: Teatro Nacional D. Maria II

- 4/6/1997 - As moscas: Alunos da ESTC

- 3/10/1998 - As troianas: Contacto - Companhia de Teatro Água Corrente

- 18/12/1998 - A respeitosa: TEP - Teatro Experimental do Porto

- 22/11/2001 - A porta fechada: TEF - Teatro Experimental do Funchal

- 22/11/2003 - As moscas: Sin-Cera

- 17/2/2008 - Huis-clos (Sem saída): Grupo de Teatro O Grito

-21/10/2009 - Huis-clos: Mala Voadora 
